\title{
EVALUATION OF THE EFFECT OF FOUR ESSENTIAL OILS AS POTENTIAL ALTERNATIVES FOR MONENSIN ON RUMEN FERMENTATION CHARACTERISTICS AND NUTRIENT DEGRADABILITY
}

\author{
Eman A. Elwakeel ${ }^{1}$, A.A. Al-Sagheer ${ }^{2}$ and Mariam G. Ahmed ${ }^{1}$ \\ ${ }^{I}$ Department of Animal and Fish Production, Faculty of Agriculture, El-Shatby, Alexandria University, \\ Egypt. \\ ${ }^{2}$ Animal Production Department, Faculty of Agriculture, Zagazig University, Zagazig, Egypt.
}

(Received 2/4/2019, accepted 27/6/2019)

\section{SUMMARY}

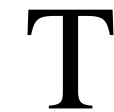

The objective of this study was to evaluate the effect of four essential oils (EOs) from guava (Psidium guajava), citronella (Cymbopogon nardus), lemongrass (Cymbopogon citratus), and geranium (Pelargonium graveolens) on gas production and rumen fermentation in vitro as a natural substitute for the ionophore antibiotic monensin. These EOs are chemically characterised by Gas Chromatography Mass and evaluated in vitro at four different concentrations $(0,15,30$, and $45 \mu \mathrm{l}$ per $45 \mathrm{ml}$ buffered rumen fluid) regarding their effects on gas production and rumen fermentation characteristics and were compared to those of monensin. Compared to the negative control, monensin significantly depressed gas production and truly degraded dry matter (TDDM) but enhanced propionate production. All EOs except $P$. graveolens significantly decreased gas production with increasing concentrations. TDDM was significantly reduced with $C$. citratus (at $45 \mu \mathrm{l}$ ) and $P$. graveolens (at 30 and $45 \mu \mathrm{l}$ ). No significant change was detected in the ammonia nitrogen concentration with all assayed EOs except $C$. nardus and C. Citrus. Compared to monensin and the negative control, C. nardus and C. Citrus reduced the ammonia concentration at high levels. High levels of all tested EOs significantly reduced protozoa counts. The EOs of $C$. citratus (at $45 \mu \mathrm{l}$ ) and P. graveolens (at 30 and $45 \mu \mathrm{l}$ ) also significantly increased the acetate proportion. Moreover, the acetate to propionate ratio was significantly increased by $30 \mu \mathrm{l} P$. graveolens. The results of the current study concluded that the tested EOs, except $P$. graveolens, efficiently diminished gas production with a similar potency to monensin. Furthermore, they exceed the monensin in their ability to reduce the ammonia nitrogen concentration and protozoa count without adversely affecting volatile fatty acid levels. But, they were less effective than monensin in modifying ruminal volatile fatty acid profile especially propionate and acetate to propionate ratio. Hence, P. guajava, C. nardus, and $C$. citratus EOs could be a safe and promising rumen manipulator.

Keywords: Essential oils, monensin, nutrient degradability and rumen fermentation.

\section{INTRODUCTION}

For decades, ionophores such as monensin have been effectively adopted as feed supplements to modulate ruminal fermentation and boost feed efficiency in livestock production (Khorrami et al., 2015; Melchior et al., 2018). Monensin may modify ruminal fermentation mainly by enhancing energy status via an antiporter action (Newbold et al., 2013) and inhibiting ruminal fungi, protozoa, and Gram-positive bacteria rather than Gram-negative bacteria (Duffield et al., 2008). The selective inhibition of Gramnegative bacteria resulted in an elevated propionate percentage and a concurrent reduction in acetate, butyrate, and methane production (Johnson et al., 2009). However, the use of ionophores such as monensin in ruminant nutrition is confronted by reduced social approval because of antimicrobial resistance in animals and humans together with the presence of residues in meat and milk, which pose a serious hazard to public health (Chesson, 2006; Yang et al., 2010). Accordingly, more regulations to prohibit their use are appearing and ruminant nutritionists are keenly trying to find suitable safe substitutes for these feed supplements (Anassori et al., 2011; Khorrami et al., 2015). 


\section{Elwakeel et al.}

Currently, the use of plant bioactive ingredients as safe feed supplements to enhance nutrient utilisation and ruminal fermentation has gained substantial attention among ruminant nutritionists (Ye et al., 2018). Essential oils (EOs) are volatile aromatic complexes generated from plants by steam and/or water distillation (Calsamiglia et al., 2007). EOs reportedly manipulate the rumen fermentation pattern (Schären et al., 2017) as a response of their antimicrobial actions against both Gram-negative and Gram-positive bacteria due to the presence of phenolic and terpenoid compounds (Dorman and Deans, 2000). Essential oils show important biological activities, which account for the development of aromatherapy used in complementary and alternative medicine. Nevertheless, to our knowledge, information about the impact of the tested EOs compared with monensin to manipulate ruminal fermentation is still scarce. The goal of the current study was to assess the inclusion effects of four EOs in different doses on the rumen fermentation profile, nutrient degradability, and gas production (GP) in vitro.

\section{MATERIALS AND METHODS}

The current work was carried out at the Advanced Laboratory of Animal Nutrition, Department of Animal and Fish Production, Faculty of Agriculture (El-Shatby), Alexandria University.

\section{Plant material and extraction of essential oils:}

Fresh leaves from citronella (Cymbopogon nardus), guava (Psidium guajava), geranium (Pelargonium graveolens) and lemongrass (Cymbopogon citratus) were gathered during the flower stage from different locations in the Alexandria governorate and El-Kanater El-Khayria city, Qalyubia Governorate, Egypt. Plant species were verified and authenticated by the book Flora of Egypt (Tackholm 1974). The plants leaves were washed with distilled water and dehydrated for five days at room temperature. A Clevenger-type apparatus was used for EO isolation by hydro distillation for $4 \mathrm{~h}$. Collected EOs were stored in amber glass bottles at $4^{\circ} \mathrm{C}$ for biological and gas chromatography-mass spectrometry (GC-MS) analyses.

\section{Analysis of essential oils using GC-MS:}

The chemical natures of the individual constituents of citronella, guava, geranium and lemongrass following dilution with hexane were identified using GC-MS (Thermo ISQ, Waltham, MA, USA). A GCMS system equipped with a TG-5MS capillary column (Thermo, P/N 26098. 1430; $30 \mathrm{~m} \times 0.32 \mathrm{~mm} \times 0.25$ $\mu \mathrm{m})$ was used. The injection volume was $1 \mu \mathrm{L}$ using an autosampler, and the injector temperatures were set at $240^{\circ} \mathrm{C}$. The carrier gas was helium at a flow rate of $1 \mathrm{~mL} / \mathrm{min}$ with a split ratio of 1:10. The initial oven temperature was $50^{\circ} \mathrm{C}$ and was maintained for $8 \mathrm{~min}$ and then raised to $130^{\circ} \mathrm{C}$ at a rate of $8^{\circ} \mathrm{C} / \mathrm{min}$. Then, the temperature was increased to $200^{\circ} \mathrm{C}$ at a rate of $5^{\circ} \mathrm{C}$ and ultimately raised to $280^{\circ} \mathrm{C}$ at $15^{\circ} \mathrm{C} / \mathrm{min}$, which was maintained for $4 \mathrm{~min}$. The ion source temperature was $230^{\circ} \mathrm{C}$, and the electron impact ionisation (ET) was $70 \mathrm{eV}$. Mass spectra were analysed in the SCAN mode over the range of 33 at $400 \mathrm{amu}$, with an emission current of 34.6 VA and electron multiplier voltage of $1392 \mathrm{~V}$.

\section{Substrate description and treatments:}

The diet used as a substrate was a total mixed ration (50\% roughage: $50 \%$ concentrate). The formulation of the basal diet was $50 \%$ berseem hay, yellow corn $20 \%$, what bran $12.5 \%$, soybean meal $5 \%$, crushed horse bean $10.75 \%$, limestone $1 \%$, salt $0.5 \%$ and a mixture of minerals and vitamins $0.25 \%$. The basal diet was milled using a Wiley mill to permit a $1 \mathrm{~mm}$ screen and was adopted as a substrate. The chemical analysis of the experimental diet was conducted for organic matter (OM), dry matter (DM), ether extract (EE), and crude protein (CP) according to the procedure of the AOAC (2006). Acid detergent fibre (ADF), neutral detergent fibre (NDF), and acid detergent lignin (ADL) were estimated following the method of Van Soest et al. (1991) with an ANKOM 220 fibre analyser (ANKOM Technology Corporation, Macedon, NY, USA). NDF analysis was performed with heat stable a-amylase. The proximate analysis of the substrate was 89.61\% OM, 17.00\% CP, 3.97\% EE, 50.63\% NDF, 20.61\% ADF, 4.34\% ADL, 16.27\% cellulose, 30.02\% hemicellulose, and $10.39 \%$ Ash. EOs from guava, citronella, lemongrass, and geranium were supplemented at four levels $(0,15,30$, and $45 \mu 1$ per $45 \mathrm{ml}$ buffered rumen fluid). In the present study, monensin (Rumensin®, Elanco, Itapira, Brazil) was used as a positive control at a concentration of $93.6 \mu \mathrm{g}$ per $300 \mathrm{mg}$ basal diet. 


\section{Inoculum donors and in vitro gas production assay:}

The inoculum donors were four adult rumen-fistulated Barki sheep with an average body weight of $46.0 \pm 1.6 \mathrm{~kg}$. Sheep were fed $0.75 \mathrm{~kg}$ as-fed concentrate feed mixture and berseem hay ad lib.head/daily. The proximate analysis of the concentrate feed mixture was $14.5,2.7,89.5,22.6$, and $38.2 \%$ for OM, CP, EE, $\mathrm{NDF}$, and ADF, respectively, on a DM basis. Before the morning feeding, liquid and solid rumen contents were obtained individually via the cannula by a stainless steel probe connected to a large volume syringe. Collected rumen contents were kept in pre-heated $\left(39^{\circ} \mathrm{C}\right)$ shielded flasks and transferred to the laboratory under anaerobic conditions. The collected rumen contents were compressed via four layers of cheese cloth and placed in a water bath at $39^{\circ} \mathrm{C}$ with $\mathrm{CO}_{2}$ saturation until inoculation.

The in vitro GP technique was conducted following the technique of Theodorou et al. (1994) with the modification of the semi-automatic system of Mauricio et al. (1999), utilising a pressure transducer in 120 $\mathrm{ml}$ serum tubes incubated for $24 \mathrm{~h}$ at $39^{\circ} \mathrm{C}$. The tested diets ( $300 \mathrm{mg}$ as-fed) were incubated with $30 \mathrm{ml} \mathrm{MB} 9$ medium and $15 \mathrm{ml}$ assorted rumen fluid. MB9 was composed of $2.8 \mathrm{~g} \mathrm{NaCl}, 0.1 \mathrm{~g} \mathrm{CaCl}_{2}, 0.1 \mathrm{~g}$ $\mathrm{MgSO}_{4} .7 \mathrm{H}_{2} \mathrm{O}, 2.0 \mathrm{~g} \mathrm{KH}_{2} \mathrm{PO}_{4}$ and $6.0 \mathrm{~g} \mathrm{Na}_{2} \mathrm{HPO}_{4}$ per litre. Then, the $\mathrm{CO}_{2}$ was rushed for $30 \mathrm{~min}$, and the $\mathrm{pH}$ was set to 6.8 (Onodera and Handerson, 1980). Bottles were sealed by rubber stoppers, agitated, and incubated at $39^{\circ} \mathrm{C}$. After 6-, 12- and 24-h incubations, the gas headspace pressure was recorded by a pressure transducer, and the bottles were shaken by hand. Based on the regression obtained in our system and conditions, the GP volume at every incubation time was estimated. For each GP assay, three GP runs were used. GP measurements were performed in triplicate for each run. Each run had six blank tubes comprising buffered rumen fluid lacking substrate, six negative control tubes with substrate only, six positive control tubes with substrate fortified monensin, and six tubes with substrate for each EO dose. GP volumes were expressed as ml per $\mathrm{g}$ incubated DM. After the end of the incubation, three bottle contents were adopted for the estimation of TDOM and TDDM based on the technique of Blümmel and Becker (1997). The NH3-N concentration was determined in another three bottles (Preston, 1995). For determining protozoal numbers, rumen fluid was mixed with methyl green-formalin saline solution and kept for later counting based on the method of Galyean (1989). Following the method of Palmquist and Conrad (1971), VFAs were estimated using a gas chromatograph (GC Thermo TRACE 1300). The VFA of known concentrations was used as a standard for calibration, and no internal standard was used.

\section{Statistical analysis:}

Because positive and negative controls were used for all treatment combinations, the design of the current study did not have a factorial arrangement. Hence, to compare EOs at different concentrations with monensin and the negative control, an analysis we performed using the four EOs with three concentrations each combined with monensin and the negative control to form one treatment variable with 14 levels $(4 \times 3$ + monensin + negative control). The proc mixed procedure of SAS (2002) was used to analyse the obtained results. The data were analysed within each sample as time (run) that considered a random effect. Significance was declared at $P<0.05$, and Dunnett's multiple comparison test (Dunnett, 1955) was used to compare the negative control, monensin and tested EOs. The contrast statement was used to determine the linear and quadratic variable response to increasing doses of EOs.

\section{RESULTS AND DISCUSSION}

\section{Composition of the tested essential oils:}

EOs extracted from diverse plant species can differ in stereochemistry, chemical structures and bioactive activities (Burt, 2004). Numerous components were recognised from the chromatograms of the tested EOs of P. guajava, C. nardus, C. citratus, and P. graveolens. As shown in Table (1), based on the relative percentages of the chromatogram areas, the chief compounds of $P$. guajava EO were cyclopentene,3-(2propenyl) (24.26\%), jasminlactone (11.10\%), lactic acid pentamethylbenzyl ester (10.8\%), and 4acetoxyquinolin-2-one (10.7\%). Citronellol (33.6\%), geraniol $(27.2 \%)$, oleic acid (2.9\%), and epoxylinalooloxide (2.4\%) were the major components of $C$. nardus EO, as shown in Table (2). As depicted in Table (3), $\beta$-citral (31.8\%) and $\alpha$-citral (25.8\%) represented the major components of $C$. citratus EO. In contrast, á-myrcene, cyclopentene, 1-butyl-, 5-hepten-2-one, 6-methyl-, $\beta$-linalool, cis-geranyl acetate, trans13-octadecenoic acid, and linoleic acid were minor components. $\alpha$ Citronellol, $\beta$ citronellol, linalool, geranyl 
formate, and caryophyllene constituted $18.3,8.41,6.98,5.16$, and 4.84\%, respectively, of P. graveolens EO chromatogram areas (Table 4).

Table (1): Main bioactive components of guava (Psidium guajava L.) essential oil detected by gas chromatography/mass spectrometry.

\begin{tabular}{|c|c|c|c|c|c|}
\hline Peak & Compounds & $\mathrm{MW}^{\dagger}$ & Formula & $\mathrm{RT}^{\dagger}$ & Area $\%$ \\
\hline $1-$ & Cyclopentene,3-(2-propenyl)- & 108 & $\mathrm{C}_{8} \mathrm{H}_{12}$ & 4.80 & 24.26 \\
\hline $2-$ & Jasminlactone & 168 & $\mathrm{C}_{10} \mathrm{H}_{16} \mathrm{O}_{2}$ & 13.88 & 11.10 \\
\hline $3-$ & Lactic acid, pentamethylbenzyl ester & 250 & $\mathrm{C}_{15} \mathrm{H}_{22} \mathrm{O}_{3}$ & 11.23 & 10.77 \\
\hline 4- & 4-Acetoxyquinolin- 2 one & 203 & $\mathrm{C}_{11} \mathrm{H}_{9} \mathrm{NO}_{3}$ & 14.23 & 10.71 \\
\hline $5-$ & $\begin{array}{l}\text { 1H-Cycloprop[e]azule ne, decahydro-1,1,7-trimethyl-4- } \\
\text { methylene-,[1aR-(1aà,4aà,7à,7aá, 7bà)] }\end{array}$ & 204 & $\mathrm{C}_{15} \mathrm{H}_{24}$ & 11.52 & 3.68 \\
\hline $6-$ & à-Caryophyllene & 204 & $\mathrm{C}_{15} \mathrm{H}_{24}$ & 11.76 & 2.42 \\
\hline 7- & $\begin{array}{l}\text { Naphthalene, } \\
\text {-methylene-7-(1-methy lethenyl)-, [4aR-(4aà,7à,8aá)]- }\end{array}$ & 204 & $\mathrm{C}_{15} \mathrm{H}_{24}$ & 12.35 & 2.21 \\
\hline $8-$ & 9-Octadecenoic acid, methyl ester, (E)- & 296 & $\mathrm{C}_{19} \mathrm{H}_{36} \mathrm{O}_{2}$ & 23.83 & 2.00 \\
\hline 9- & Hexadecanoic acid, methyl ester & 270 & $\mathrm{C}_{17} \mathrm{H}_{34} \mathrm{O}_{2}$ & 20.66 & 1.95 \\
\hline $10-$ & $\begin{array}{l}\text { Azulene, 1,2,3,3a,4,5,6,7-octahydro-1,4-dimethyl-7-(1- } \\
\text { methylethenyl)-, [1R-(1à,3aá,4à,7á)]- }\end{array}$ & 204 & $\mathrm{C}_{15} \mathrm{H}_{24}$ & 12.50 & 1.93 \\
\hline $12-$ & à-Cadinol & 222 & $\mathrm{C}_{15} \mathrm{H}_{26} \mathrm{O}$ & 15.50 & 1.80 \\
\hline $13-$ & $\begin{array}{l}\text { Naphthalene, } \\
\text { dimethyl-4-(1-methyle thyl)-, (1S-cis)- }\end{array}$ & 202 & $\mathrm{C}_{15} \mathrm{H}_{22}$ & 13.00 & 1.60 \\
\hline $14-$ & $\begin{array}{l}\text { Cyclopentane, } \\
\text { 1-isopropenyl-3-methyl 2-(1,1-dicyanomethyl)- }\end{array}$ & 188 & $\mathrm{C}_{12} \mathrm{H}_{16} \mathrm{~N}_{2}$ & 14.54 & 1.42 \\
\hline $15-$ & Ylangene & 204 & $\mathrm{C}_{15} \mathrm{H}_{24}$ & 10.41 & 1.37 \\
\hline 16- & Caryophyllene & 204 & $\mathrm{C}_{15} \mathrm{H}_{24}$ & 11.88 & 1.37 \\
\hline $17-$ & $\begin{array}{l}\text { 12-Oxabicyclo[9.1.0]dodeca-3,7-diene, } \\
\text { tetramethyl-, [1R-1R*,3E,7E,11R*)] }\end{array}$ & 220 & $\mathrm{C}_{15} \mathrm{H}_{24} \mathrm{O}$ & 14.65 & 1.23 \\
\hline $18-$ & Ledol & 222 & $\mathrm{C}_{15} \mathrm{H}_{26} \mathrm{O}$ & 14.35 & 1.05 \\
\hline
\end{tabular}

Table (2): Main bioactive components of citronella (Cymbopogon nardus) essential oil detected by gas chromatography/mass spectrometry.

\begin{tabular}{llllll}
\hline Peak & Compounds & $\mathrm{MW}^{\dagger}$ & $\mathrm{Formula}^{\dagger}$ & $\mathrm{RT}^{\dagger}$ & Area\% $^{\dagger}$ \\
\hline $1-$ & Citronellol & 156 & $\mathrm{C}_{10} \mathrm{H}_{20} \mathrm{O}$ & 8.40 & 33.55 \\
$2-$ & Geraniol & 153 & $\mathrm{C}_{10} \mathrm{H}_{19} \mathrm{~N}$ & 8.97 & 27.21 \\
$3-$ & Oleic Acid & 282 & $\mathrm{C}_{18} \mathrm{H}_{34} \mathrm{O}_{2}$ & 24.65 & 2.85 \\
$4-$ & Epoxy-linalooloxide & 186 & $\mathrm{C}_{10} \mathrm{H}_{18} \mathrm{O}_{3}$ & 10.44 & 2.40 \\
$5-$ & Hexadecanoic acid, methyl ester & 270 & $\mathrm{C}_{17} \mathrm{H}_{34} \mathrm{O}_{2}$ & 20.71 & 2.19 \\
$6-$ & 11-Octadecenoic acid, methyl ester & 296 & $\mathrm{C}_{19} \mathrm{H}_{36} \mathrm{O}_{2}$ & 23.87 & 2.17 \\
$7-$ & Linalool & 154 & $\mathrm{C}_{10} \mathrm{H}_{18} \mathrm{O}$ & 5.84 & 2.11 \\
$8-$ & 5-Hepten-2-one, 6-methyl- & 126 & $\mathrm{C}_{8} \mathrm{H}_{14} \mathrm{O}$ & 4.05 & 1.87 \\
$9-$ & 3-(4,8,12-Trimethyltridecyl) furan & 292 & $\mathrm{C}_{20} \mathrm{H}_{36} \mathrm{O}$ & 9.85 & 1.62 \\
$10-$ & Erucic acid & 338 & $\mathrm{C}_{22} \mathrm{H}_{42} \mathrm{O}_{2}$ & 28.14 & 1.41 \\
$12-$ & cis-Verbenol & 152 & $\mathrm{C}_{10} \mathrm{H}_{16} \mathrm{O}$ & 7.21 & 0.91 \\
$13-$ & Octadecanoic acid & 284 & $\mathrm{C}_{18} \mathrm{H}_{36} \mathrm{O}_{2}$ & 24.90 & 0.75 \\
$14-$ & n-Hexadecanoic acid & 256 & $\mathrm{C}_{16} \mathrm{H}_{32} \mathrm{O}_{2}$ & 21.42 & 0.71 \\
$15-$ & 8-Hydroxycarvotanace tone & 168 & $\mathrm{C}_{10} \mathrm{H}_{16} \mathrm{O}_{2}$ & 10.25 & 0.66 \\
$16-$ & Geranyl acetate & 196 & $\mathrm{C}_{12} \mathrm{H}_{20} \mathrm{O}_{2}$ & 10.55 & 0.57 \\
$17-$ & 2-Tridecanone & 198 & $\mathrm{C}_{13} \mathrm{H}_{26} \mathrm{O}$ & 12.50 & 0.51 \\
$18-$ & cis-Verbenol & 152 & $\mathrm{C}_{10} \mathrm{H}_{16} \mathrm{O}$ & 6.91 & 0.50 \\
$19-$ & Citronellal & 154 & $\mathrm{C}_{10} \mathrm{H}_{18} \mathrm{O}$ & 6.71 & 0.39 \\
$20-$ & Octadecanoic acid, methyl ester & 298 & $\mathrm{C}_{19} \mathrm{H}_{38} \mathrm{O}_{2}$ & 24.31 & 0.31 \\
\hline
\end{tabular}

${ }^{\top} M W=$ molecular weight, $R T=$ retention time, minutes. 
Table (3): Main bioactive components of lemongrass (Cymbopogon citratus) essential oil detected by gas chromatography/mass spectrometry.

\begin{tabular}{llllcc}
\hline Peak & Compounds & $\mathrm{MW}^{\dagger}$ & Formula & $\mathrm{RT}^{\dagger}$ & Area\% \\
\hline $1-$ & $\beta$-Citral & 152 & $\mathrm{C}_{10} \mathrm{H}_{16} \mathrm{O}$ & 8.91 & 31.86 \\
$2-$ & $\alpha$-Citral & 152 & $\mathrm{C}_{10} \mathrm{H}_{16} \mathrm{O}$ & 8.36 & 25.82 \\
$3-$ & a-Myrcene & 136 & $\mathrm{C}_{10} \mathrm{H}_{16}$ & 4.11 & 4.11 \\
$4-$ & Cyclopentene, 1-butyl- & 124 & $\mathrm{C}_{9} \mathrm{H}_{16}$ & 8.60 & 3.90 \\
$5-$ & 5-Hepten-2-one, 6-methyl- & 126 & $\mathrm{C}_{16} \mathrm{H}_{14} \mathrm{O}$ & 4.04 & 2.12 \\
$6-$ & $\beta$-Linalool & 154 & $\mathrm{C}_{10} \mathrm{H}_{18} \mathrm{O}$ & 5.83 & 1.86 \\
$7-$ & cis-Geranyl acetate & 196 & $\mathrm{C}_{12} \mathrm{H}_{20} \mathrm{O}_{2}$ & 10.55 & 1.81 \\
$8-$ & trans-13-Octadecenoic acid & 282 & $\mathrm{C}_{18} \mathrm{H}_{34} \mathrm{O}_{2}$ & 24.55 & 1.57 \\
$9-$ & Linoleic acid & 280 & $\mathrm{C}_{18} \mathrm{H}_{32} \mathrm{O}_{2}$ & 24.45 & 1.48 \\
$10-$ & 1-(Cyclopropyl-nitro-methyl)-cyclopentanol & 185 & $\mathrm{C}_{9} \mathrm{H}_{15} \mathrm{NO}_{3}$ & 10.42 & 1.23 \\
$12-$ & (Z)-Geranic acid & 196 & $\mathrm{C}_{12} \mathrm{H}_{20} \mathrm{O}_{2}$ & 20.19 & 1.15 \\
$13-$ & Methyl 10,11-tetradecadienoate & 238 & $\mathrm{C}_{15} \mathrm{H}_{26} \mathrm{O}_{2}$ & 9.83 & 1.03 \\
\hline
\end{tabular}

${ }^{T} M W=$ molecular weight, $R T=$ retention time, minutes .

Table (4): Main bioactive components of geranium (Pelargonium graveolens) essential oil detected by gas chromatography/mass spectrometry.

\begin{tabular}{llllll}
\hline Peak & Compounds & $\mathrm{MW}^{\dagger}$ & Formula & $\mathrm{RT}^{\dagger}$ & Area\% \\
\hline $1-$ & $\alpha$ Citronellol & 156 & $\mathrm{C}_{10} \mathrm{H}_{20} \mathrm{O}$ & 8.20 & 18.28 \\
$2-$ & $\beta$ Citronellol & 156 & $\mathrm{C}_{10} \mathrm{H}_{20} \mathrm{O}$ & 8.63 & 8.41 \\
$3-$ & Linalool & 182 & $\mathrm{C}_{11} \mathrm{H}_{18} \mathrm{O}_{2}$ & 8.85 & 6.98 \\
$4-$ & Geranyl formate & 182 & $\mathrm{C}_{11} \mathrm{H}_{18} \mathrm{O}_{2}$ & 6.96 & 5.16 \\
$5-$ & Caryophyllene & 204 & $\mathrm{C}_{15} \mathrm{H}_{24}$ & 14.94 & 4.84 \\
$8-$ & Geranyl tiglate & 236 & $\mathrm{C}_{15} \mathrm{H}_{24} \mathrm{O}_{2}$ & 16.43 & 2.21 \\
$9-$ & $\delta$-Cadinene & 204 & $\mathrm{C}_{15} \mathrm{H}_{24}$ & 13.05 & 2.14 \\
$10-$ & trans-13-Octadecenoic acid & 282 & $\mathrm{C}_{18} \mathrm{H}_{34} \mathrm{O}_{2}$ & 24.58 & 2.07 \\
$12-$ & ë-Neoclovene & 204 & $\mathrm{C}_{15} \mathrm{H}_{24}$ & 11.20 & 1.94 \\
$13-$ & $\beta$-Bourbonene & 204 & $\mathrm{C}_{15} \mathrm{H}_{24}$ & 10.61 & 1.67 \\
$14-$ & Hexadecanoic acid, methyl ester & 270 & $\mathrm{C}_{17} \mathrm{H}_{34} \mathrm{O}_{2}$ & 20.17 & 1.38 \\
& 8-Octadecenoic acid, methyl ester & 296 & $\mathrm{C}_{19} \mathrm{H}_{36} \mathrm{O}_{2}$ & 23.87 & 1.35 \\
$15-$ & D-Limonene & 136 & $\mathrm{C}_{10} \mathrm{H}_{16}$ & 4.67 & 1.09 \\
$16-$ & Geranyl propionate & 210 & $\mathrm{C}_{13} \mathrm{H}_{22} \mathrm{O}_{2}$ & 12.12 & 1.14 \\
$17-$ & Germacrene D & 204 & $\mathrm{C}_{15} \mathrm{H}_{24}$ & 12.28 & 1.11 \\
$18-$ & Geraniol butyrate & 224 & $\mathrm{C}_{14} \mathrm{H}_{24} \mathrm{O}_{2}$ & 13.71 & 1.10 \\
\hline
\end{tabular}

$M W=$ molecular weight, $R T=$ retention time, minutes.

\section{Effects on gas production and feed degradability:}

Herein, monensin was chosen as a positive control treatment due to its favourable roles in modulating ruminal fermentation. Table (5) demonstrates the effect of the tested EOs at different concentrations compared to that of monensin on both GP and feed degradability. A significant reduction in truly degraded organic matter (TDOM) and GP, expressed as $\mathrm{mL} / \mathrm{g}$ dry matter (DM) $(P=0.001)$ and $\mathrm{mL} / \mathrm{g}$ TDOM $(P=0.039)$, was obvious following the addition of monensin compared to that following the addition of the negative control. These results are in agreement with those of earlier in vitro trials (Callaway et al., 1997; Shen et al., 2017). The reduction in GP following monensin treatment is highly linked to its lipophilic nature, which causes a disturbance in the intracellular $\mathrm{K}^{+}$and $\mathrm{Na}^{+}$balance, which is injurious to Grampositive bacteria that produce higher amounts of hydrogen and lactate (Russell and Strobel, 1988). The suppressive action of monensin on feed degradability is mostly due to its cellulolytic bacteria inhibition, which is the main contributor to fibre degradability in the rumen (Narvaez et al., 2013; Jeyanathan et al., 2014). 


\section{Elwakeel et al.}

Additionally, a tendency comparable to the effect of monensin on GP (mL/g DM and mL/g TDOM) was observed for P. guajava, C. nardus, and C. citratus EOs (Table 5). Compared with the negative control, EOs achieved the maximum reduction in GP at 30 and $45 \mu \mathrm{l}$; however, there were no significant differences $(\mathrm{mL} / \mathrm{g} \mathrm{DM})$ between the EO and monensin groups. Moreover, compared to the negative control, $P$. graveolens EO did not elicit a significant change in GP. However, this decline is not detected for minor doses of these EOs and not for all added doses of EOs from both P. guajava and C. nardus.

Table (5): Effect of different levels of essential oils (EOs) on gas production and feed degradability after a 24-h in vitro incubation.

\begin{tabular}{|c|c|c|c|c|c|}
\hline \multirow[b]{2}{*}{ Additive } & \multirow{2}{*}{$\begin{array}{l}\text { Dosage } \\
{[\mu \mathrm{l} / 45 \mathrm{ml} \text { rumen fluid] }}\end{array}$} & \multicolumn{2}{|c|}{ Gas production } & \multirow[b]{2}{*}{$\begin{array}{l}\text { TDDM } \\
(\mathrm{g} / 100 \mathrm{~g})\end{array}$} & \multirow[b]{2}{*}{$\begin{array}{l}\text { TDOM } \\
(\mathrm{g} / 100 \mathrm{~g})\end{array}$} \\
\hline & & {$[\mathrm{ml} / \mathrm{g} \mathrm{DM}]$} & {$[\mathrm{ml} / \mathrm{g}$ TDOM] } & & \\
\hline Negative control & & $124.47 \dagger$ & $181.60 \dagger$ & $69.61 \dagger$ & 69.95 \\
\hline Monensin & & $93.64 *$ & $107.67 *$ & $66.56 *$ & 67.36 \\
\hline \multirow[t]{5}{*}{ Psidium guajava } & 15 & $111.04 \dagger$ & $156.21 \dagger$ & $70.26 \dagger$ & $71.17 \dagger$ \\
\hline & 30 & $104.73 *$ & $148.43 * \dagger$ & $69.80 \dagger$ & $70.52 \dagger$ \\
\hline & 45 & $92.39 *$ & $135.54 * \dagger$ & 67.15 & 68.13 \\
\hline & Linear & 0.140 & 0.015 & 0.109 & 0.138 \\
\hline & Quadratic & $<0.001$ & $<0.001$ & 0.027 & 0.172 \\
\hline \multirow[t]{5}{*}{ Cymbopogon nardus } & 15 & $103.40 *$ & $149.00 * \dagger$ & 68.70 & 69.42 \\
\hline & 30 & $94.95 *$ & $140.61 * \dagger$ & 67.00 & 67.69 \\
\hline & 45 & $89.09 *$ & $133.69 *$ & 67.40 & 67.96 \\
\hline & Linear & 0.005 & 0.003 & 0.472 & 0.704 \\
\hline & Quadratic & $<0.001$ & $<0.001$ & 0.055 & 0.103 \\
\hline \multirow[t]{5}{*}{$\begin{array}{l}\text { Cymbopogon } \\
\text { citratus }\end{array}$} & 15 & $114.89 \dagger$ & $161.78 \dagger$ & $70.31 \dagger$ & $71.08 \dagger$ \\
\hline & 30 & $103.68 *$ & $151.12 * \dagger$ & 68.47 & 69.15 \\
\hline & 45 & $95.75^{*}$ & $142.13 * \dagger$ & $66.64 *$ & 67.47 \\
\hline & Linear & 0.316 & 0.09 & 0.196 & 0.127 \\
\hline & Quadratic & $<0.001$ & 0.001 & 0.002 & 0.006 \\
\hline \multirow[t]{5}{*}{$\begin{array}{l}\text { Pelargonium } \\
\text { graveolens }\end{array}$} & 15 & $118.60 \dagger$ & $171.31 \dagger$ & 68.40 & 69.17 \\
\hline & 30 & 111.64 & $161.85 \dagger$ & $66.89 *$ & 68.60 \\
\hline & 45 & 108.44 & $151.34 \dagger$ & $66.85^{*}$ & 68.19 \\
\hline & Linear & 0.571 & 0.553 & 0.304 & 0.472 \\
\hline & Quadratic & 0.049 & 0.013 & 0.011 & 0.061 \\
\hline \multicolumn{6}{|l|}{ Statistics } \\
\hline SEM & & 3.43 & 4.21 & 0.66 & 0.59 \\
\hline$P$-value & & $<0.001$ & $<0.001$ & 0.001 & 0.039 \\
\hline
\end{tabular}

*Means within a column that have a symbol are significantly different $(P<0.05)$ from the control.

$\dagger$ Means within a column that have a symbol are significantly different $(P<0.05)$ from monensin.

$D M=$ dry matter; SEM = standard error of the mean; TDDM= truly degraded dry matter; TDOM = truly degraded organic matter.

The decrease in GP with increasing concentrations of EOs detected in the current study is consistent with the findings of Patra and Yu (2012) and Sallam et al. (2011) who also reported a linear decrease in total GP with increasing EO doses. Notably, the reduction in GP associated with a reduction in the protozoa count observed here may be linked to the antimicrobial activity of EOs because of their active components (Calsamiglia et al., 2007). For instance, citral, the main active ingredient detected in C. citratus herein, significantly reduces GP in the rumen with notable antimicrobial action (Lin et al., 2013). Additionally, geraniol, a major component of $C$. nardus, exerts antimicrobial action against both Gram-negative and 
Gram-positive bacteria (Dorman and Deans, 2000) and reduces total GP when tested at $500 \mathrm{mg}^{-1}$ (Pirondini et al., 2015).

Compared to the negative control, EOs from C. citratus $(45 \mu \mathrm{l})$ and P. graveolens (30 and $45 \mu \mathrm{l})$ decreased truly degraded dry matter (TDDM), but no significant difference was observed between these EOs and monensin (Table 5). Digestibility reduction is the outcome of the emulation between digestion and passage rates (Van Soest, 1994). In numerous reports, adding blended EOs reduced both the effective degradability and ruminal degradation rate of some protein supplements (Newbold et al., 2004). In the current study, $C$. citratus markedly decreased TDDM together with GP. The antimicrobial activity of its major component citral could be responsible for the decline in TDDM detected in the current experiment. The hostile effect of $P$. graveolens on TDDM may be due to citronellol as it reportedly efficiently hinders rumen microbial activity and exhibits activity against both Gram-negative and Gram-positive bacteria (Wan et al., 1998; Burt, 2004).

\section{Effects on rumen fermentation products:}

Generally, compared to the negative control, monensin did not elicit any significant alterations in the $\mathrm{NH}_{3}-\mathrm{N}$ concentration, $\mathrm{pH}$, and protozoa count (Table 6). All concentrations of the tested EOs did not alter $(P=0.141)$ the ruminal $\mathrm{pH}$.

Table (6): Effect of different levels of essential oils (EOs) on the ammonia concentration and protozoal count after a 24-h in vitro incubation.

\begin{tabular}{|c|c|c|c|c|}
\hline Additive & $\begin{array}{l}\text { Dosage } \\
\text { [ } \mu \mathrm{l} / 45 \mathrm{ml} \text { rumen fluid] }\end{array}$ & $\begin{array}{l}\mathrm{NH}_{3}-\mathrm{N} \\
(\mathrm{mg} / 100 \mathrm{~mL})\end{array}$ & $\mathrm{pH}$ & $\begin{array}{l}\text { Protozoa } \\
\left(10^{5} / \mathrm{mL}\right)\end{array}$ \\
\hline Negative control & & 27.95 & 5.76 & 6.80 \\
\hline Monensin & & 29.53 & 5.62 & 6.10 \\
\hline \multirow{5}{*}{ Psidium guajava } & 15 & 27.50 & 5.61 & 6.10 \\
\hline & 30 & 25.29 & 5.55 & 5.10 \\
\hline & 45 & $22.41 \dagger$ & 5.55 & $4.40 *$ \\
\hline & Linear & 0.834 & 0.526 & 0.583 \\
\hline & Quadratic & 0.027 & 0.361 & 0.007 \\
\hline \multirow[t]{5}{*}{ Cymbopogon nardus } & 15 & 27.22 & 5.59 & 5.70 \\
\hline & 30 & 24.10 & 5.63 & 4.90 \\
\hline & 45 & $20.88^{* \dagger}$ & 5.66 & $3.30 * \dagger$ \\
\hline & Linear & 0.894 & 0.450 & 0.586 \\
\hline & Quadratic & 0.007 & 0.839 & 0.005 \\
\hline \multirow[t]{5}{*}{ Cymbopogon citratus } & 15 & 27.00 & 5.57 & 5.60 \\
\hline & 30 & $23.66 \dagger$ & 5.60 & 5.20 \\
\hline & 45 & $22.45 \dagger$ & 5.67 & $4.00^{*}$ \\
\hline & Linear & 0.848 & 0.356 & 0.399 \\
\hline & Quadratic & 0.030 & 0.845 & 0.018 \\
\hline \multirow[t]{5}{*}{ Pelargonium graveolens } & 15 & 26.44 & 5.60 & 5.90 \\
\hline & 30 & 25.68 & 5.62 & 5.10 \\
\hline & 45 & $23.81 \dagger$ & 5.63 & $4.60 *$ \\
\hline & Linear & 0.643 & 0.470 & 0.363 \\
\hline & Quadratic & 0.066 & 0.704 & 0.010 \\
\hline \multicolumn{5}{|l|}{ Statistics } \\
\hline SEM & & 0.50 & 0.04 & 0.18 \\
\hline$P$-value & & 0.027 & 0.141 & 0.021 \\
\hline
\end{tabular}

*Means within a column that have a symbol are significantly different $(P<0.05)$ from the control.

+ Means within a column that have a symbol are significantly different $(P<0.05)$ from monensin.

$S E M=$ standard error of the mean. 


\section{Elwakeel et al.}

In the current study, compared to both negative and positive controls, EO from C. nardus (45 $\mu 1)$ significantly decreased the $\mathrm{NH}_{3}-\mathrm{N}$ concentration. Compared to monensin, the four tested EOs at a concentration of $45 \mu \mathrm{l}$ significantly reduced the $\mathrm{NH}_{3}-\mathrm{N}$ concentration (Table 6). A similar trend was previously noted with other EOs, such as clove oil (Gunal et al., 2013), eugenol (Busquet et al., 2006; Castillejos et al., 2006), Eucalyptus citriodora, and Ocimum gratissimum (Kouazounde et al., 2015). However, according to Castillejos et al. (2008), $500 \mathrm{mg} / \mathrm{l}$ clove oil had no influence on $\mathrm{NH}_{3}-\mathrm{N}$ concentrations. The small effects of $P$. guajava, $P$. graveolens and $C$. citratus EOs on the $\mathrm{NH}_{3}-\mathrm{N}$ concentration may be due to the collaboration between their active components and proteins (Gunal et al., 2013). Nonetheless, the strong suppressive effect of $C$. nardus EO on ammonia production could probably result from the reduction in hyper-ammonia producing bacteria such as Peptostreptococcus anaerobius and Clostridium sticklandii due to the antimicrobial activity of its major component geraniol (McEwan et al., 2002). These changes lead to further inhibition of deamination of amino acids and subsequently decreased ammoniagenesis (Castillejos et al., 2006).

Table (7): Effect of different levels of essential oils (EOs) on the volatile fatty acid concentration (mM) after a 24-h in vitro incubation.

\begin{tabular}{|c|c|c|c|c|c|c|c|}
\hline \multirow[b]{2}{*}{ Additive } & \multirow{2}{*}{$\begin{array}{l}\text { Dosage } \\
{[\mu \mathrm{l} / 45 \mathrm{ml}} \\
\text { rumen fluid] }\end{array}$} & \multicolumn{4}{|c|}{ Volatile fatty acids (mM) } & \multirow{2}{*}{$\begin{array}{l}\mathrm{C} 2 / \mathrm{C} 3 \\
\text { ratio }\end{array}$} & \multirow{2}{*}{ Total } \\
\hline & & $\begin{array}{l}\text { Acetate } \\
\text { (C2) }\end{array}$ & $\begin{array}{l}\text { Propionate } \\
\text { (C3) }\end{array}$ & Butyrate & Valerate & & \\
\hline Negative control & & 36.99 & $11.02 \dagger$ & 9.08 & 1.03 & 3.38 & 58.13 \\
\hline Monensin & & 43.84 & $15.90 *$ & 8.81 & 0.92 & 2.77 & 69.47 \\
\hline \multirow[t]{5}{*}{ Psidium guajava } & 15 & 41.79 & 10.54 & 8.71 & 0.45 & 3.98 & 61.47 \\
\hline & 30 & 43.51 & 12.78 & 10.93 & 0.92 & 3.45 & 68.14 \\
\hline & 45 & 44.74 & 11.24 & 8.59 & 1.03 & 4.04 & 65.60 \\
\hline & Linear & 0.105 & 0.862 & 0.872 & 0.269 & 0.230 & 0.199 \\
\hline & Quadratic & 0.094 & 0.185 & 0.700 & 0.394 & 0.839 & 0.059 \\
\hline \multirow[t]{5}{*}{ Cymbopogon nardus } & 15 & 38.97 & 10.47 & 6.64 & 0.54 & 3.74 & 58.61 \\
\hline & 30 & 45.29 & 13.30 & 9.18 & 1.06 & 3.43 & 68.83 \\
\hline & 45 & 40.84 & 13.19 & 7.79 & 0.64 & 3.14 & 62.47 \\
\hline & Linear & 0.173 & 0.947 & 0.934 & 0.398 & 0.128 & 0.375 \\
\hline & Quadratic & 0.070 & 0.024 & 0.728 & 0.910 & 0.045 & 0.058 \\
\hline \multirow[t]{5}{*}{ Cymbopogon citratus } & 15 & 41.48 & 10.57 & 9.68 & 0.41 & 4.03 & 62.14 \\
\hline & 30 & 40.91 & 10.72 & 11.95 & 0.62 & 3.91 & 64.20 \\
\hline & 45 & $48.89 *$ & 10.16 & 9.23 & 0.93 & $4.84 \dagger$ & 69.20 \\
\hline & Linear & 0.065 & 0.749 & 0.354 & 0.161 & 0.172 & 0.230 \\
\hline & Quadratic & 0.001 & 0.644 & 0.657 & 0.625 & 0.025 & 0.028 \\
\hline \multirow[t]{5}{*}{ Pelargonium graveolens } & 15 & 41.94 & 12.60 & 7.97 & 0.66 & 3.37 & 63.15 \\
\hline & 30 & $49.81 *$ & 9.12 & 9.36 & 1.04 & $5.52 * \dagger$ & 69.32 \\
\hline & 45 & $48.71 *$ & 10.98 & 7.49 & 0.67 & $4.67 \dagger$ & 67.84 \\
\hline & Linear & 0.002 & 0.592 & 0.783 & 0.487 & 0.342 & 0.031 \\
\hline & Quadratic & $<0.001$ & 0.089 & 0.850 & 0.935 & 0.004 & 0.005 \\
\hline \multicolumn{8}{|l|}{ Statistics } \\
\hline SEM & & 1.01 & 0.72 & 0.87 & 0.06 & 0.12 & 1.59 \\
\hline$P$-value & & 0.004 & 0.001 & 0.813 & 0.377 & $<0.001$ & 0.067 \\
\hline
\end{tabular}

Compared with the negative control, monensin significantly enhanced propionate $(P=0.001)$ but not acetate production. These results are in agreement with those of earlier in vitro trials (Callaway et al., 1997; Shen et al., 2017). In the rumen, propionate is generated by the succinate or acrylate pathway, and the 
succinate pathway is the predominant pathway (Jeyanathan et al., 2014). Monensin reportedly boosts propionate production via the succinate pathway by increasing propionate and succinate producer abundance and reducing non-producers (Schären et al., 2017).

As mirrored by VFA profiles, the current study showed that compared to no supplementation, supplementation with EOs from $C$. citratus $(45 \mu \mathrm{l})$ and $P$. graveolens $(30$ and $45 \mu \mathrm{l})$ significantly increased acetate production (Table 7). Similarly, Kamalak et al. (2011) reported that 200-1200 $\mathrm{mg} \mathrm{L}^{-1}$ Citrus sinensis EO altered the VFA profile by increasing acetate. Additionally, in the present study, the acetate to propionate ratio increased with increasing levels of $P$. graveolens EO, most likely due to molecular hydrogen accumulation. Here, the effects exhibited by $P$. graveolens on VFA are consistent with the results of Patra and $\mathrm{Yu}$ (2012) who found no alteration in TVFA level with M. piperita, whereas both acetate and the acetate to propionate ratio increased. Conversely, Canaes et al. (2017) reported that citral oil reduced the ruminal acetate proportion and acetate to propionate ratio. This discrepancy could be due to the variation in the concentration and activity of secondary metabolites within a given EO source.

In summary, the tested EOs manipulated the rumen fermentation characteristics but to varying extents. Differences in the properties and chemical structures of EOs might underlie these discrepancies. All tested EOs, except $P$. graveolens, efficiently diminished gas production with a similar potency to monensin. Furthermore, they exceed the monensin in their ability to reduce the ammonia nitrogen concentration and protozoa count without adversely affecting volatile fatty acid levels. But, they were less effective than monensin in modifying ruminal volatile fatty acid profile especially propionate and acetate to propionate ratio. Hence, P. guajava, $C$. nardus, and $C$. citratus EOs could be a safe and promising rumen manipulator.

\section{REFERENCES}

Anassori, E., B. Dalir-Naghadeh, R. Pirmohammadi, A. Taghizadeh, S. Asri-Rezaei, M. Maham, S. Farahmand-Azar, and P. Farhoomand (2011). Garlic: A potential alternative for monensin as a rumen modifier. Livest. Sci., 142(1-3):276-287.

AOAC (2006). Official Methods of Analysis, $18^{\text {th }}$ ed., Washington, DC, USA.

Blümmel, M., and K. Becker (1997). The degradability characteristics of fifty-four roughages and roughage neutral-detergent fibres as described by in vitro gas production and their relationship to voluntary feed intake. Br. J. Nutr., 77(5):757-768.

Burt, S. (2004). Essential oils: their antibacterial properties and potential applications in foods-a review. Int. J. Food Microbiol., 94(3):223-253.

Busquet, M., S. Calsamiglia, A. Ferret, and C. Kamel (2006). Plant extracts affect in vitro rumen microbial fermentation. J. Dairy Sci., 89(2):761-771.

Callaway, T. R., A. M. C. De Melo, and J. B. Russell (1997). The effect of nisin and monensin on ruminal fermentations in vitro. Curr. Microbiol., 35(2):90-96.

Calsamiglia, S., M. Busquet, P. Cardozo, L. Castillejos, and A. Ferret (2007). Invited review: essential oils as modifiers of rumen microbial fermentation. J. Dairy Sci., 90(6):2580-2595.

Canaes, T. S., F. Zanferari, B. L. Maganhe, C. S. Takiya, T. H. Silva, T. A. Del Valle, and F. P. Rennó (2017). Increasing dietary levels of citral oil on nutrient total tract digestibility, ruminal fermentation, and milk composition in Saanen goats. Anim. Feed Sci. Technol., 229:47-56.

Castillejos, L., S. Calsamiglia, and A. Ferret (2006). Effect of essential oil active compounds on rumen microbial fermentation and nutrient flow in in vitro systems. J. Dairy Sci., 89(7):2649-2658.

Castillejos, L., S. Calsamiglia, J. Martin-Tereso, and H. Ter Wijlen (2008). In vitro evaluation of effects of ten essential oils at three doses on ruminal fermentation of high concentrate feedlot-type diets. Anim. Feed Sci. Technol., 145(1-4):259-270.

Chesson, A. (2006). Phasing out antibiotic feed additives in the EU: worldwide relevance for animal food production. Antimicrobial growth promoters-where do we go from here:69-81. 


\section{Elwakeel et al.}

Dorman, H., and S. G. Deans (2000). Antimicrobial agents from plants: antibacterial activity of plant volatile oils. J. Appl. Microbiol., 88(2):308-316.

Dunnett, C. W. (1955). A multiple comparison procedure for comparing several treatments with a control. J. Am. Stat. Assoc., 50(272): 1096-1121.

Duffield, T., A. Rabiee, and I. Lean (2008). A meta-analysis of the impact of monensin in lactating dairy cattle. Part 1. Metabolic effects. J. Dairy Sci., 91(4):1334-1346.

Francis, G., Z. Kerem, H. P. Makkar, and K. Becker (2002). The biological action of saponins in animal systems: a review. Br. J. Nutr., 88(6):587-605.

Galyean, M. (1989). Laboratory procedure in animal nutrition research. Department of Animal and Life Science. New Mexico State University, USA:188.

Gunal, M., A. Ishlak, and A. Abughazaleh (2013). Evaluating the effects of six essential oils on fermentation and biohydrogenation in in vitro rumen batch cultures. Czech J. Anim. Sci., 58:243-252.

Jeyanathan, J., C. Martin, and D. Morgavi (2014). The use of direct-fed microbials for mitigation of ruminant methane emissions: a review. Animal 8(2):250-261.

Johnson, M., A. Devine, J. Ellis, A. Grunden, and V. Fellner (2009). Effects of antibiotics and oil on microbial profiles and fermentation in mixed cultures of ruminal microorganisms. J. Dairy Sci., 92(9):4467-4480.

Kamalak, A., A. Atalay, C. Ozkan, A. Tatliyer, and E. Kaya (2011). Effect of essential orange (Citrus sinensis L.) oil on rumen microbial fermentation using in vitro gas production technique. J. Anim. Plant Sci., 21(4):764-769.

Khorrami, B., A. Vakili, M. D. Mesgaran, and F. Klevenhusen (2015). Thyme and cinnamon essential oils: Potential alternatives for monensin as a rumen modifier in beef production systems. Anim. Feed Sci. Technol., 200:8-16.

Kouazounde, J. B., L. Jin, F. M. Assogba, M. A. Ayedoun, Y. Wang, K. A. Beauchemin, T. A. McAllister, and J. D. Gbenou (2015). Effects of essential oils from medicinal plants acclimated to Benin on in vitro ruminal fermentation of Andropogon gayanus grass. J. Sci. Food Agric., 95(5):1031-1038.

Lin, B., J. Wang, Y. Lu, Q. Liang, and J. Liu (2013). In vitro rumen fermentation and methane production are influenced by active components of essential oils combined with fumarate. J. Anim. Physiol. Anim. Nutr., 97(1):1-9.

Mauricio, R. M., F. L. Mould, M. S. Dhanoa, E. Owen, K. S. Channa, and M. K. Theodorou (1999). A semiautomated in vitro gas production technique for ruminant feedstuff evaluation. Anim. Feed Sci. Technol., 79(4):321-330.

McEwan, N., R. Graham, R. Wallace, R. Losa, P. Williams, and C. Newbold (2002). Effect of essential oils on ammonia production by rumen microbes. Reprod. Nutr. Dev., 42(1):S65-S65.

Melchior, E., K. Hales, A. Lindholm-Perry, H. Freetly, J. Wells, C. Hemphill, T. Wickersham, J. Sawyer, and P. Myer (2018). The effects of feeding monensin on rumen microbial communities and methanogenesis in bred heifers fed in a drylot. Livest. Sci., 212:131-136.

Narvaez, N., Y. Wang, Z. Xu, and T. McAllister (2013). Effects of California chaparral plants on in vitro ruminal fermentation of forage and concentrate diet. J. Sci. Food Agric., 93(3):550-559.

Newbold, C., F. McIntosh, P. Williams, R. Losa, and R. Wallace (2004). Effects of a specific blend of essential oil compounds on rumen fermentation. Animal feed science and technology 114(1-4):105-112.

Newbold, C. J., R. J. Wallace, and N. D. Walker-Bax (2013). Potentiation by metal ions of the efficacy of the ionophores, monensin and tetronasin, towards four species of ruminal bacteria. FEMS Microbiol. Lett., 338(2):161-167.

Palmquist, D., and H. Conrad (1971). Origin of plasma fatty acids in lactating cows fed high grain or high fat diets. J. Dairy Sci., 54(7):1025-1033.

Patra, A. K., and Z. Yu (2012). Effects of essential oils on methane production, fermentation, abundance and diversity of rumen microbial populations. Appl. Environ. Microbiol., AEM. 00309-00312. 
Pirondini, M., S. Colombini, L. Malagutti, L. Rapetti, G. Galassi, R. Zanchi, and G. M. Crovetto (2015). Effects of a selection of additives on in vitro ruminal methanogenesis and in situ and in vivo NDF digestibility. Anim. Sci. J., 86(1):59-68.

Preston, T. (1995). Tropical animal feeding: a manual for research workers. Rome: FAO. p. 191-264.

Russell, J., and H. Strobel (1988). Effects of additives on in vitro ruminal fermentation: a comparison of monensin and bacitracin, another gram-positive antibiotic. Journal of animal science 66(2):552-558.

Sallam, S., and A. Abdalla (2011). Antimethanogenic and antiprotozoal effect of some essential oils in vitro. Egy. J. Anim. Prod 48:203-215.

Sallam, S. M., S. A. Abdelgaleil, I. C. da Silva Bueno, M. E. Nasser, R. C. Araujo, and A. L. Abdalla (2011). Effect of some essential oils on in vitro methane emission. Arch. Anim. Nutr., 65(3):203-214.

Schären, M., C. Drong, K. Kiri, S. Riede, M. Gardener, U. Meyer, J. Hummel, T. Urich, G. Breves, and S. Dänicke (2017). Differential effects of monensin and a blend of essential oils on rumen microbiota composition of transition dairy cows. J. Dairy Sci., 100(4):2765-2783.

Shen, J., Z. Liu, Z. Yu, and W. Zhu (2017). Monensin and nisin affect rumen fermentation and microbiota differently in vitro. Front. Microbiol. 8:1111.

Theodorou, M. K., B. A. Williams, M. S. Dhanoa, A. B. McAllan, and J. France (1994). A simple gas production method using a pressure transducer to determine the fermentation kinetics of ruminant feeds. Anim. Feed Sci. Technol., 48(3-4):185-197.

Ushida, K., and J. Jouany (1996). Methane production associated with rumen-ciliated protozoa and its effect on protozoan activity. Lett. Appl. Microbiol., 23(2):129-132.

van Nevel, C., and D. Demeyer (1988). Manipulation of rumen fermentation. In: Hobson PN, editor. The rumen microbial ecosystem. Elsevier Applied Science: London.387-443.

Van Soest, P. (1994). Nutritional ecology of the ruminant., 2nd edn.(Cornell University Press: Ithaca, NY). Google Scholar

Van Soest, P. v., J. Robertson, and B. Lewis (1991). Methods for dietary fiber, neutral detergent fiber, and nonstarch polysaccharides in relation to animal nutrition. J. Dairy Sci., 74(10):3583-3597.

Wan, J., A. Wilcock, and M. Coventry (1998). The effect of essential oils of basil on the growth of Aeromonas hydrophila and Pseudomonas fluorescens. J. Appl. Microbiol., 84(2):152-158.

Yang, W., B. Ametaj, C. Benchaar, M. He, and K. Beauchemin (2010). Cinnamaldehyde in feedlot cattle diets: intake, growth performance, carcass characteristics, and blood metabolites. J. Anim. Sci., 88(3):1082-1092.

Ye, D., S. K. R. Karnati, B. Wagner, J. L. Firkins, M. L. Eastridge, and J. M. Aldrich (2018). Essential oil and monensin affect ruminal fermentation and the protozoal population in continuous culture. J. Dairy Sci., 101(6):5069-5081. 


\title{
Elwakeel et al.
}

\section{تقييم تأثير أربعة أنواع من الزيوت العطرية كبدائل محتملة للمونينسين على خصائص التخمر ومعدل تحلل}

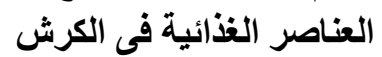

\author{
إيمان على الوكيل1 ، أدهم عبدالله الصغير² و مريم جمال أحمد1 \\ 1 قسم الإنتاج الحيواني والسكي ـ كلية الزراعة (الشاطبي) - جامعة الإسكندرية - الإسكندريةـ مصر. \\ 2قسم الإنتاج الحيوانس ـ كلية الزراعة - جامعة الزقازيق- مصر.
}

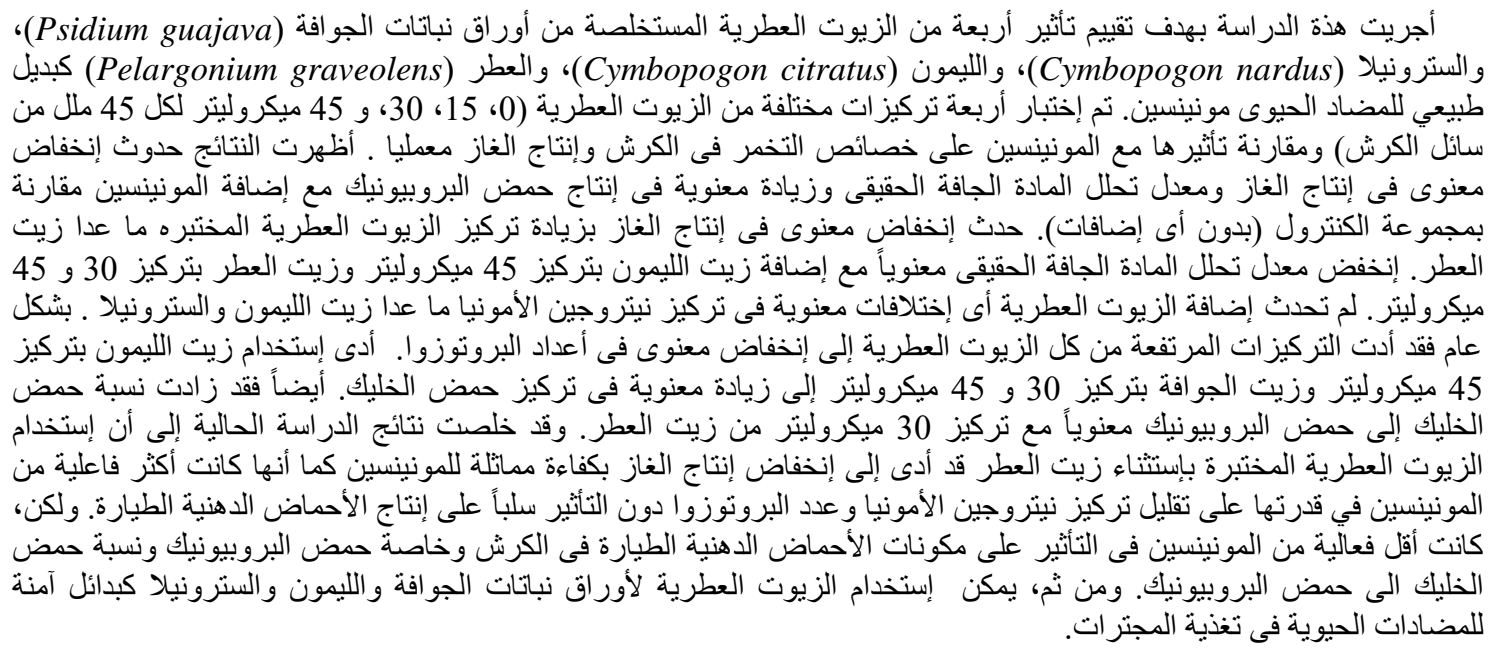

